\title{
Qualification sanitaire des troupeaux, représentations du risque selon les acteurs et les disciplines
}

\author{
Christian Ducrot ${ }^{1}$, Dominique Pécaud ${ }^{2, \star}$, Étienne Petit ${ }^{3}$, Stéphane Krebs ${ }^{4}$, Anne-France Viet ${ }^{5}$, \\ Benoit Durand $^{6}$, Fabienne Biteau-Coroller ${ }^{7}$, François Beaudeau ${ }^{8}$, Brigitte Frappat ${ }^{9}$, \\ Didier Calavas ${ }^{10}$, Christine Fourichon ${ }^{8}$
}

1 Vétérinaire épidémiologiste, INRA, UR346 Épidémiologie animale, 63122 Saint-Genès-Champanelle, France

2 Sociologue, Institut de l'homme et de la technologie, École polytechnique de l'Université de Nantes, 44306 Nantes cedex 3 , France

3 Vétérinaire conseil, Fédération régionale des groupements de défense sanitaire de Bourgogne, 42 rue de Mulhouse, 21000 Dijon, France

4 Économiste, INRA, ENVN, UMR1300 Bio-agression, épidémiologie et analyse de risque, La Chantrerie, BP 40706, 44307 Nantes cedex 3, France

5 Modélisatrice, INRA, ENVN, UMR1300 Bio-agression, épidémiologie et analyse de risque, La Chantrerie, BP 40706, 44307 Nantes cedex 3, France

6 Vétérinaire modélisateur, AFSSA, LERPAZ (Laboratoire d'études et de recherches en pathologie animale et zoonoses), Unité d'épidémiologie, 27-31 avenue du Général Leclerc, 94701 Maisons-Alfort cedex, France

7 Vétérinaire épidémiologiste, CIRAD, Département Environnement et Sociétés, UR AGIR (Animal et gestion intégrée des risques), Campus international de Baillarguet, 34398 Montpellier cedex 5, France

8 Vétérinaires épidémiologistes, INRA, ENVN, UMR1300 Bio-agression, épidémiologie et analyse de risque, La Chantrerie, BP 40706, 44307 Nantes cedex 3, France

9 Agronome, Institut de l'élevage, service Ingénierie de projets, 149 rue de Bercy, 75012 Paris, France

10 Vétérinaire épidémiologiste, AFSSA Lyon, Unité épidémiologie, 31 avenue Tony Garnier, 69364 Lyon cedex 07, France

Rédigé par une dizaine d'auteurs, cet article associe des chercheurs de différentes disciplines et des praticiens autour de la notion de qualification sanitaire des animaux et des troupeaux à propos des maladies infectieuses non règlementées par les pouvoirs publics. Il débouche sur des considérations en termes de risque qui ne se déclinent pas dans les mêmes termes selon les disciplines, ni selon qu'on est vendeur ou acheteur des animaux ... Cet article est l'un des premiers publié dans la revue qui soit issu des travaux conduits dans le cadre du programme Agriculture et Développement durable (ADD), lancé en 2005 à l'initiative des établissements de recherche, Inra, Cirad et Cemagref, et de l'ANR.

La Rédaction

Auteur correspondant : C. Ducrot, ducrot@clermont.inra.fr

* D. Pécaud est aussi chercheur au Centre d'étude et de recherche sur les risques et les vulnérabilités (CERReV, MRSH-Caen) et chercheur associé à l'unité Socio-économie, Environnement, Développement (SEED, Université de Liège). 


\section{Mots-clés :}

qualification sanitaire ;

élevage ; interdisciplinarité; coconstruction ; risque

\section{Keywords:}

sanitary

qualification;

farm;

interdisciplinarity; coconstruction; risk
La qualification sanitaire vise à établir de manière objective et fiable le statut sanitaire d'un animal ou d'un ensemble d'animaux au regard d'une maladie infectieuse. Concevoir une qualification sanitaire repose sur des connaissances biologiques concernant l'agent pathogène, ses voies de transmission et les moyens de détection de la maladie. Les modèles mathématiques et les outils statistiques et probabilistes permettent d'intégrer ces connaissances tout en prenant en compte l'incertitude et la variabilité des données biologiques. Les sciences humaines apportent un éclairage sur les enjeux, les perceptions et les logiques des individus et des collectifs, afin d'étudier la demande et l'acceptabilité de la qualification sanitaire. Chaque discipline apporte ainsi son point de vue sur la notion de risque sous-jacente à la démarche de qualification. Cette approche interdisciplinaire nécessite une coconstruction de la recherche, qui doit dépasser les différences culturelles et épistémologiques entre disciplines. Elle n'obéit pas à un seul type de rationalité, scientifique ou juridico-administrative; elle mobilise nécessairement des connaissances savantes et des savoirs empiriques et dépend de nombreuses décisions pouvant engendrer convergences ou contradictions.

\begin{abstract}
Sanitary qualification of farm animals and risk representations by the stakeholders and scientific disciplines. The aim of sanitary qualification of farm animals is to implement collective strategies to control the spread of infectious diseases. More specifically, it seeks to establish objectively and accurately the status of an animal or animal population, which will be used for animal exchange and trading, with regard to a given infectious disease. The approach is based upon biological knowledge about the infectious agent including infection process and detection means. In addition, mathematical modelling, statistics and probability tools allow integrating these pieces of knowledge as well as taking into account uncertainty and the variability of biological parameters. Finally, Social and Human sciences, and particularly economics and sociology provide an understanding of stakes, social representations, and points of view of individuals and groups, in order to study the demand and acceptability of sanitary qualification for a given infectious disease. In this approach, each discipline contributes its own standpoint on the underlying question of risk. This interdisciplinary approach requires co-constructing the research topic and stepping beyond epistemological and cultural differences between disciplines. Furthermore, designing a qualification results from a collective social construction. It does not solely obey rationality based on science and regulation needs; it includes necessarily both scientific and common knowledge and is dependant upon various political decisions that can lead to consensus or contradiction.
\end{abstract}

\section{Introduction}

La qualification sanitaire d'animaux de rente s'inscrit dans une démarche de lutte collective contre les maladies infectieuses. Elle doit permettre aux élevages d'être protégés contre les risques d'infection liés à l'introduction d'animaux, mais également de s'assurer que les produits animaux issus des élevages soient indemnes d'infections zoonotiques. Dans le cas des maladies dites réglementées, la qualification sanitaire peut être décidée par les pouvoirs publics, en raison des enjeux économiques (fièvre aphteuse) ou de santé publique (tuberculose) liés à ces maladies. Les enjeux du commerce international, découlant d'éventuelles restrictions liées à une qualification, sont cruciaux dans les décisions, les dispositifs visant alors à garantir que des pays ou régions sont indemnes (Zepeda et al., 2005; Domenech et al., 2006). Pour des maladies non réglementées et présentes sur les territoires (dites endémiques), des dispositifs de qualification sanitaire peuvent être mis en place volontairement par les acteurs dès lors que l'impact économique d'une maladie justifie, selon eux, une action collective (diarrhée virale bovine, par exemple).

D'un point de vue technique, la qualification sanitaire vise à établir de manière objective et fiable le statut sanitaire d'un animal ou d'un ensemble d'animaux au regard $\mathrm{d}^{\prime}$ une maladie transmissible. Pour concevoir un système de qualification, il est nécessaire de prendre en compte les caractéristiques de la maladie, notamment ses modalités de transmission, de même que les modalités de diffusion de l'agent pathogène dans l'organisme et les moyens de détection de la maladie. Par ailleurs, il faut également considérer le contexte d'utilisation de la qualification (systèmes d'élevage concernés, risques de transmission, réglementation) et évaluer les résultats attendus sur différents critères d'intérêt, à différents horizons de temps et pour les différents acteurs impliqués.

Le programme de recherche $\mathrm{ACDUQ}^{1}$ a été mis en œuvre pour produire des bases de conception et des méthodes d'évaluation de dispositifs de qualification sanitaire en élevage de ruminants. Impliquant une communauté d'une quinzaine de scientifiques et experts de terrain issus de disciplines variées (biologie, épidémiologie, modélisation, sociologie, économie), ce projet a permis de poser les bases méthodologiques d'une réflexion interdisciplinaire concernant la qualification sanitaire. Les travaux ont porté sur quatre agents infectieux présents

\footnotetext{
1 ACDUQ : Action collective pour une maîtrise durable de la santé animale : qualification sanitaire en élevage de ruminants. Il s'agit d'un programme financé par l'ANR (appel d'offres «Agriculture pour le développement durable 2005 »); cet article en est issu.
} 
de façon endémique dans les régions d'étude : Coxiella burnetii, bactérie responsable de la fièvre $Q$, le mycoplasme de la péripneumonie contagieuse bovine (PPCB) ${ }^{2}$, le virus de la diarrhée virale bovine (BVD) ${ }^{3}$ et l'agent de la tremblante du mouton ${ }^{4}$. Quatre enquêtes ont été menées sur la base d'entretiens individuels et collectifs, qui ont été réalisés auprès de cent vingt éleveurs bovins et d'une vingtaine d'acteurs intervenant dans les élevages.

Le présent article se propose de montrer en quoi la conception d'une qualification sanitaire pertinente relève d'une complémentarité d'approches entre sciences humaines, biologiques et mathématiques. Après une brève présentation des objectifs et des enjeux de la qualification sanitaire, chacune de ces approches sera abordée pour montrer son apport dans la construction du dispositif, notamment en abordant de points de vue différents la notion de risque qui est sous-jacente à une réflexion sur la qualification sanitaire. Le propos est illustré par les travaux conduits sur les maladies étudiées. Pour finir, une analyse socioanthropologique de l'expérience du collectif de recherche impliqué dans le projet ACDUQ permettra $\mathrm{d}$ 'aborder la question de la construction interdisciplinaire de la qualification sanitaire.

\section{Objectifs et enjeux de la qualification sanitaire}

La qualification sanitaire (Toma et al., 1996) a pour objectif d'établir de manière objective et fiable le statut sanitaire d'un animal ou d'un ensemble d'animaux (troupeau, zone géographique) au regard d'une maladie donnée, souvent infectieuse ou contagieuse. Le but essentiel de la qualification sanitaire est de fournir une garantie au regard de cette maladie dans le cadre d'échanges ou de rassemblements d'animaux ${ }^{5}$. Ce raisonnement découle

\footnotetext{
${ }^{2}$ La PPCB est la maladie bovine transfrontalière la plus répandue en Afrique; très contagieuse, elle est responsable de mortalités, de pertes en lait et en force de travail.

${ }^{3}$ La BVD est une infection virale contagieuse très répandue, responsable de baisses de production laitière, de mortalité des jeunes et de dégradation des performances de reproduction et de croissance.

${ }^{4}$ La tremblante du mouton est une encéphalopathie spongiforme transmissible, contagieuse, toujours fatale, sans traitement ni vaccin.

${ }^{5}$ L'idée est de garantir que l'animal vendu ou l'élevage qui vend les animaux est indemne de telle maladie au moment de la vente; à part des situations particulières (par exemple statut non-IPI [animaux infectés permanents] pour la BVD), le vendeur n'est plus responsable en cas d'infection après la vente. En effet, le caractère contagieux et souvent ubiquiste des maladies infectieuses ne permet pas d'engager la responsabilité du vendeur au-delà de la vente. Ce point est particulièrement important lorsque celle-ci fait intervenir un rassemblement d'animaux qui ne présenteraient pas de garanties sanitaires.
}

du fait que nombre de maladies infectieuses sont véhiculées par des animaux porteurs de l'agent pathogène, et que la distinction entre animaux sains et infectés par le biais d'une qualification sanitaire devient dès lors un moyen de maîtrise de la propagation de la maladie. La qualification sanitaire d'un animal donné s'appuie souvent sur le statut établi à l'échelle du troupeau ou de la zone géographique dont il est issu. Pour une maladie contagieuse, il est en effet plus fiable d'établir ce statut à partir d'un échantillon d'animaux. Les protocoles de qualification sanitaire comportent habituellement la démonstration de l'absence de la maladie ou de l'agent pathogène en question et la mise en place de mesures de protection visant à contrôler le risque d'introduction de la maladie. En matière de recherche, différents travaux ont porté sur la mise au point d'outils statistiques destinés à apporter un niveau de garantie de l'absence de maladie dans une population (Cannon et Roe, 1982; Cameron et Baldock, 1998 ; Johnson et al., 2003).

Peu de maladies font l'objet d'une qualification sanitaire. La décision de mettre en œuvre une qualification à l'échelle d'une collectivité d'élevages procède d'une combinaison de raisons relatives à l'intérêt et à la faisabilité, par essence très variable selon la maladie et son impact global. Les enjeux liés à la qualification peuvent concerner la santé publique (fièvre Q) ou la santé animale seule (BVD, PPCB). La faisabilité technique de la qualification conditionne pour une large part son existence et les protocoles retenus; elle dépend non seulement des outils techniques disponibles (prélèvements et analyses), mais aussi des connaissances biologiques sur la maladie (pathogénie et épidémiologie). Enfin, contribuent de manière importante les enjeux économiques de la qualification, au regard des coûts de la maladie mais aussi des impacts commerciaux de la qualification, qui peut ouvrir ou fermer des marchés. C'est ainsi qu'au-delà de l'objectif initial de préserver des infections les élevages sains, la qualification sanitaire est aussi une arme économique de protection de marchés. Ces différentes composantes impliquées dans la mise en œuvre d'une qualification sanitaire sont complexes et en constante interrelation, d'où la nécessité de les identifier précisément ainsi que leur articulation. Par exemple, les performances des tests de dépistage auront une incidence majeure sur le coût de la qualification, mais aussi sur la qualité de la garantie offerte.

La notion de qualification sanitaire est étroitement associée à celle de "garantie sanitaire », qui sous-entend la notion de risque, pour les échanges notamment : risque par défaut, c'est-à-dire une garantie insuffisante qui autorise la qualification et l'échange d'animaux contaminés ( «risque acheteur ») ou risque par excès, c'est-à-dire la déqualification abusive d'un élevage sain, qui se voit privé d'accès à un marché, voire éliminé dans le cadre d'une mesure réglementaire («risque vendeur»). Les sources d'erreurs relèvent de l'imperfection des tests ou 
du protocole de qualification, mais aussi de la méconnaissance de la maladie ou d'une mauvaise application du protocole sur le terrain. Selon les enjeux, il est plus ou moins nécessaire de rassurer les acteurs concernés sur la politique sanitaire menée autour de la qualification. C'est l'objet des démarches de «certification » au sens large réalisées par des tiers, comme le vétérinaire ou un laboratoire (attestation, certificat), un organisme privé certificateur $\left(\mathrm{ACERSA}^{6}\right.$ ) ou l'État pour les maladies réglementées. Dans le cadre des échanges internationaux, ce sont les règles énoncées par $1^{\prime} \mathrm{OIE}^{7}$ qui servent souvent de référence.

Une qualification sanitaire semble d'autant plus facile à mettre en œuvre qu'elle répond à un enjeu économique justifié au regard de son coût, qu'elle est applicable dans les conditions du terrain et qu'elle est fiable. En pratique, toutes ces conditions sont rarement remplies et des compromis doivent être trouvés. Pour répondre à ces critères, le dispositif de qualification doit reposer sur un socle de connaissances biologiques solides : connaissances sur l'agent pathogène et ses voies de transmission, la pathogénie de la maladie, la réaction des animaux à l'infection, les moyens de détection des animaux infectés. Notre propos n'est pas ici de développer plus avant la démarche en biologie (somme toute classique), mais d'aborder les autres bases sur lesquelles une qualification sanitaire est envisagée.

\section{L'apport des mathématiques et de la modélisation}

Un des éléments majeurs dans la construction d'une qualification sanitaire tient à l'incertitude et à la variabilité des données biologiques. Par ailleurs, intégrer les connaissances biologiques (dose infectante, probabilité d'infection) pour en déduire les conséquences en termes de risque d'infection d'un élevage n'est pas trivial. Les modèles mathématiques et les outils statistiques et probabilistes permettent d'intégrer ces composantes dans la conception d'une qualification sanitaire.

\section{Risque vendeur, approches probabilistes et acquisition de la qualification}

Déterminer le statut sanitaire d'un troupeau passe en général par des tests biologiques sur les animaux, visant à détecter l'agent lui-même ou des marqueurs de l'infection (anticorps). Néanmoins, il n'existe pas de test parfait (il y a des résultats faussement positifs et d'autres faussement négatifs), et les tests ne portent généralement que sur des échantillons d'animaux. Des approches probabilistes

6 ACERSA : Association de certification de la santé animale.

7 OIE : Office international des épizooties. permettent de quantifier la probabilité d'erreur dans le dispositif de qualification. La démarche habituelle est de définir un niveau de risque accepté (prévalence ${ }^{8}$ cible) et de calculer la probabilité que le troupeau testé présente une prévalence supérieure à ce seuil, compte tenu de la taille de l'échantillon, des performances des tests et de leurs résultats. Si cette probabilité est faible (en général $<5 \%$ ), l'élevage est considéré comme indemne. La validité de cette conclusion repose sur une définition adéquate de la prévalence cible, en général fixée à une valeur assez basse par rapport à la prévalence usuellement observée dans les élevages atteints pour permettre un niveau de garantie important.

Dans le cadre du projet ACDUQ, ce raisonnement a été mis en œuvre pour étudier des protocoles de qualification sanitaire vis-à-vis de la tremblante du mouton, dont les particularités ont amené à développer une approche spécifique. Les tests de dépistage actuels (sur encéphale) ne peuvent être pratiqués que sur des animaux morts et la maladie n'est détectable qu'à l'âge adulte; aussi le nombre d'animaux qu'il est possible de tester est-il borné par le nombre d'animaux adultes morts ou abattus pour la consommation. Par ailleurs, le déterminisme génétique fort de la sensibilité individuelle se traduit par le fait qu'un résultat d'analyse négatif obtenu sur un animal d'un génotype résistant apporte un niveau de preuve beaucoup moins élevé en faveur de l'absence de la maladie dans l'élevage que si ce résultat a été obtenu sur un animal d'un génotype sensible. L'approche probabiliste classique a donc été étendue afin de prendre en compte l'hétérogénéité de la sensibilité génétique à la maladie (Martinez et al., à paraître). Les résultats obtenus montrent que qualifier un élevage prend de nombreuses années si les animaux testés sont choisis au hasard. Au contraire, en privilégiant les individus génétiquement les plus sensibles, le nombre d'animaux à tester et les délais sont fortement réduits. Dans la pratique, il est rare que l'on ait l'information génétique nécessaire pour tous les animaux. En revanche la généalogie du troupeau est souvent connue de façon plus ou moins complète. Si le génotype d'une partie des animaux est connu, des algorithmes élaborés par les généticiens permettent alors de dériver des distributions de probabilités pour les génotypes de l'ensemble des animaux de la généalogie. Une seconde extension a ainsi été développée pour étudier des protocoles de qualification sanitaire vis-à-vis de la tremblante du mouton utilisant de façon optimale l'ensemble des informations disponibles sur les génotypes, les résultats de tests de dépistage et la généalogie du troupeau (Durand et al., 2009). Les résultats permettent également de calculer le coût de la qualification et d'adapter le protocole de qualification aux particularités de chaque élevage.

\footnotetext{
${ }^{8}$ La prévalence (ou plus exactement le taux de prévalence) est la proportion d'individus atteints dans une population à un moment donné.
} 
Dans la mesure où les élevages candidats à une qualification peuvent être considérés comme a priori indemnes, ces approches statiques, fondées sur l'estimation de la probabilité, à un moment donné, qu'un élevage soit infecté, s'intéressent donc plutôt au risque vendeur (probabilité de refuser la qualification à un élevage indemne).

\section{Risque acheteur, modélisation et maintien de la qualification}

Les approches probabilistes statiques ne sont par contre pas adaptées dès lors que l'on s'intéresse au risque acheteur (probabilité qu'un élevage qualifié ne soit pas indemne). D'autres approches sont alors nécessaires, qui prennent en compte la dynamique de propagation de l'infection dans un élevage et la façon dont cette infection peut ou non être détectée et au bout de combien de temps. Si l'expérimentation, en impliquant un dispositif lourd, permet d'observer la propagation de l'infection sur un nombre réduit d'animaux, les résultats ne sont pas extrapolables aux conditions de terrain. Aussi, les approches de modélisation consistant à définir une représentation simplifiée du système réel prennent tout leur intérêt pour simuler la dynamique d'une infection.

Simplifier la réalité tout en conservant les paramètres pertinents dans le modèle relève $d^{\prime}$ une démarche interdisciplinaire menée entre modélisateurs et biologistes. Les connaissances biologiques permettent $\mathrm{d}$ 'abord $\mathrm{d}$ 'identifier les caractéristiques principales du système réel à prendre en compte, de même que les moyens d'observation de la réalité (test biologique ou clinique) à inclure dans le modèle pour évaluer les protocoles de qualification. Ce travail implique les pathologistes, les zootechniciens et les modélisateurs. Ces derniers réalisent ensuite la formalisation du système, dont les résultats sont discutés avec les biologistes pour des affinements successifs. Les modalités de transmission des agents pathogènes et les outils d'observation varient selon les maladies, de même que la dynamique de la population d'animaux d'élevage à considérer et l'objectif de la modélisation. Aussi le modèle est-il défini pour répondre à une question précise dans un contexte biologique donné.

À titre d'exemple, pour comprendre la propagation du virus de la BVD dans un troupeau et estimer le délai entre début d'infection et détection de la circulation du virus au sein du troupeau par des tests, il a été développé dans le projet ACDUQ une approche de modélisation incluant la dynamique du processus de contagion au cours du temps et la variabilité des paramètres biologiques. Pour le modèle de propagation en troupeau de bovins allaitants, il s'agissait d'évaluer le niveau d'infection dans un élevage et le risque d'infection induit pour les autres élevages (Viet, 2008). Le modèle pour la propagation en troupeau bovin laitier sert à évaluer des stratégies de qualification et de maîtrise, en intégrant différentes dynamiques de
Encadré 1. Modélisation de la diffusion et du dépistage de la diarrhée virale bovine (BVD)

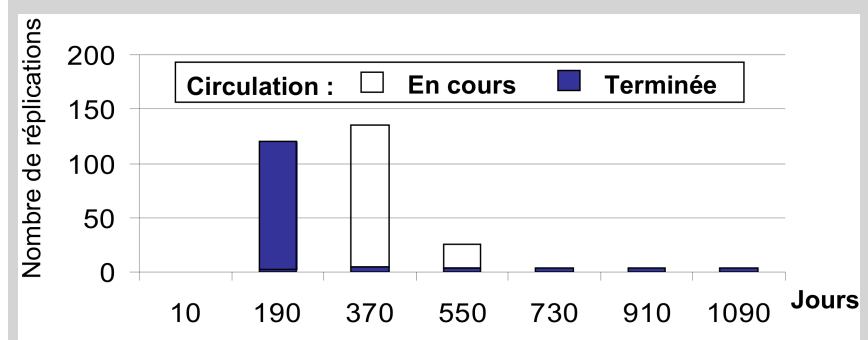

Délai entre introduction du virus et détection de sa circulation dans le troupeau

La diarrhée virale bovine (BVD) est une maladie contagieuse des bovins, non transmissible à l'homme, qui entraîne des baisses de production laitière et la dégradation des performances de reproduction et de croissance. Le virus se propage $d^{\prime}$ un troupeau à un autre principalement par contact entre bovins au pâturage ou par achat de bovins infectés, qui ne présentent pas nécessairement de signes de maladie. Un modèle mathématique a été conçu pour comprendre la propagation du virus de la BVD au sein d'un troupeau laitier et estimer le délai entre le début d'infection du troupeau et la détection de la circulation du virus au sein de ce troupeau.

À partir de ce modèle, il a été simulé le délai de détection de la circulation du virus de la BVD en nombre de jours après son introduction dans un troupeau laitier (600 simulations). Dans cet exemple, le premier test Elisa sur lait de tank est réalisé 10 jours après l'introduction d'une génisse non-IPI gestante d'un fœtus IPI (vêlage 20 jours après l'introduction). Dans 330 simulations sur 600, l'infection n'est pas détectée, ce qui est essentiellement dû à une très faible et courte épidémie dans le troupeau. Parmi les autres simulations, l'infection est détectée au bout de 6 mois dans $45 \%$ des cas, mais il peut arriver, dans de rares situations, qu'elle ne le soit qu'au bout de deux ans, voire plus, et après la fin de la circulation active du virus.

propagation, d'une très faible circulation du virus à la persistance de l'infection sur plusieurs années (Viet et al., 2004) [Encadré 1]. L'analyse de sensibilité du modèle a mis en évidence les paramètres influençant fortement les résultats, pour lesquels une bonne connaissance de leur valeur biologique est nécessaire (Ezanno et al., 2007; Ezanno et al., 2008). Pour un dispositif de qualification du troupeau vis-à-vis de la BVD à partir de tests sur le lait de mélange du troupeau, la variabilité de l'intervalle de temps entre l'introduction du virus dans le troupeau et sa détection a été simulée. La modélisation a aussi permis de déterminer un âge minimal pour l'établissement d'une garantie non-IPI ${ }^{9}$ des génisses laitières dans les troupeaux laitiers à très forte probabilité d'être indemnes de BVD (Ezanno, 2006).

\footnotetext{
${ }^{9}$ Les animaux infectés permanents immunotolérants (IPI) représentent une source très importante d'infection; ils ne fabriquent pas d'anticorps et ne peuvent donc pas être dépistés par test sérologique.
} 


\section{Approche économique et sociologique de la qualification sanitaire}

La possibilité de maîtrise de l'infection via la qualification est nécessaire mais non suffisante à la réussite de cette dernière. Le dispositif proposé doit de plus répondre à un besoin socioéconomique justifié au regard du coût de la qualification et être applicable dans les conditions du terrain.

\section{Le point de vue économique}

L'approche biotechnique de la qualification sanitaire repose sur une hypothèse implicite : les dispositifs proposés sont pleinement adoptés et mis en place par les éleveurs. Or, dans les faits, les mesures préconisées ne sont jamais mises en œuvre comme telles. Tout d'abord, la qualification sanitaire peut être abordée d'un point de vue économique, comme un outil de gestion des risques liés à l'introduction d'un agent infectieux au sein d'un troupeau, à l'occasion de l'achat d'un animal, par exemple, car elle donne une information sur le statut sanitaire de cet animal. Or, la production d'une telle information a un coût pour les parties engagées dans la transaction (Chymis et al., 2007 ; Rat-Aspert et al., 2008). Individuellement, un éleveur vendeur n'est incité à s'inscrire dans une démarche de qualification que s'il peut escompter retirer de l'information produite un bénéfice supplémentaire lors de la vente de ses animaux (ou de la commercialisation de produits animaux issus de son troupeau). Par ailleurs, les éleveurs acheteurs (ou les firmes de l'aval de la filière) ne seront enclins à privilégier des troupeaux qualifiés que s'ils en tirent un bénéfice, notamment en termes de réduction des risques.

Ensuite, dans la perspective d'une gestion collective des maladies animales, la qualification sanitaire devrait permettre de réduire la propagation de l'agent infectieux entre troupeaux, afin de limiter les externalités négatives induites par la non-maîtrise de l'agent pathogène par certains agents économiques - cela, dans le contexte où les éleveurs seraient naturellement enclins à chercher à bénéficier des effets positifs d'un programme collectif de maîtrise, sans en supporter les coûts (adoption de comportements opportunistes). Les enjeux liés à la qualification reposent ainsi sur la production à moindre coût d'une information fiable concernant le statut sanitaire d'un animal (ou d'un troupeau), de nature à garantir une adhésion des éleveurs à la stratégie de maîtrise préconisée. Lorsque le libre jeu des mécanismes de marché ne permet pas de s'assurer d'un tel résultat, la mise en place de mesures incitatives (Wolf, 2005) ou de dispositions réglementaires (Centner et Wetzstein, 1987) doit être envisagée.

\section{Le point de vue sociologique}

Une large palette d'acteurs (Fig.) est concernée par la qualification sanitaire, chacun ayant ses propres priorités, points de vue, attentes, stratégies. La connaissance de ces éléments et la compréhension des mécanismes qui les influencent sont essentielles à la mise en place d'un dispositif de qualification acceptable par une majorité d'acteurs (Kling-Éveillard, 2001). La sociologie interactionniste contribue à analyser cette réalité complexe, notamment à travers la prise en compte des logiques d'action individuelles ou collectives. De plus, elle peut participer de façon plus opérationnelle à la coconstruction des projets de qualification sanitaire.

Une étude sur les attentes en matière de qualification sanitaire, conduite en 2006 auprès des différents acteurs de la filière, a mis en avant la diversité de leurs points de vue. Les transformateurs ne sont pas toujours demandeurs d'un système sophistiqué de qualification. Ils auraient dans ce cas à gérer des circuits de produits distincts selon le niveau de qualification des «élevages sources » et à faire face à des contraintes et à des coûts supplémentaires peu susceptibles d'être répercutés ou justifiés au niveau des consommateurs. Les citoyens considèrent la qualité sanitaire des produits alimentaires comme un dû ; mais, en tant que consommateurs, ils peuvent exiger la maîtrise des risques à coût nul pour eux. Les éleveurs ont des attentes qui dépendent de l'orientation de leur élevage (lait ou viande) et des contraintes que pourrait induire la mise en place d'un nouveau dispositif de suivi sanitaire. Par exemple, en élevage laitier, un prélèvement de lait suffit pour dépister la BVD ou la fièvre $Q$, alors qu'en production de viande, il n'existe pas d'autre alternative que d'effectuer des prélèvements sanguins individuels coûteux et contraignants à réaliser. Intervient aussi la stratégie commerciale des éleveurs, à l'échelle individuelle (vente ou achat d'animaux reproducteurs à haute valeur génétique, pour lesquels des garanties sanitaires élevées sont nécessaires) ou régionale (volonté de conquérir ou de conforter des débouchés par la vente d'animaux garantis indemnes). Joue aussi la sensibilité que ces animaux présentent aux risques sanitaires, notamment aux zoonoses, plus développée en production hors sol où les pratiques de biosécurité sont déjà bien implantées. Audelà des logiques institutionnelles, chaque individu est aussi porteur d'enjeux de valeur ou de rôle professionnel qu'il exprime sous la forme de justifications précises ou de représentations plus générales influençant de façon consciente ou non ses pratiques.

Dans le projet de recherche ACDUQ, des études ont été menées auprès des éleveurs de bovins à propos des enjeux de qualification sanitaire dans le cas de la fièvre $Q$ et de la BVD. Des entretiens individuels ont été réalisés pour mieux cerner les attitudes des éleveurs vis-à-vis de la santé du troupeau, des maladies, des règles collectives 


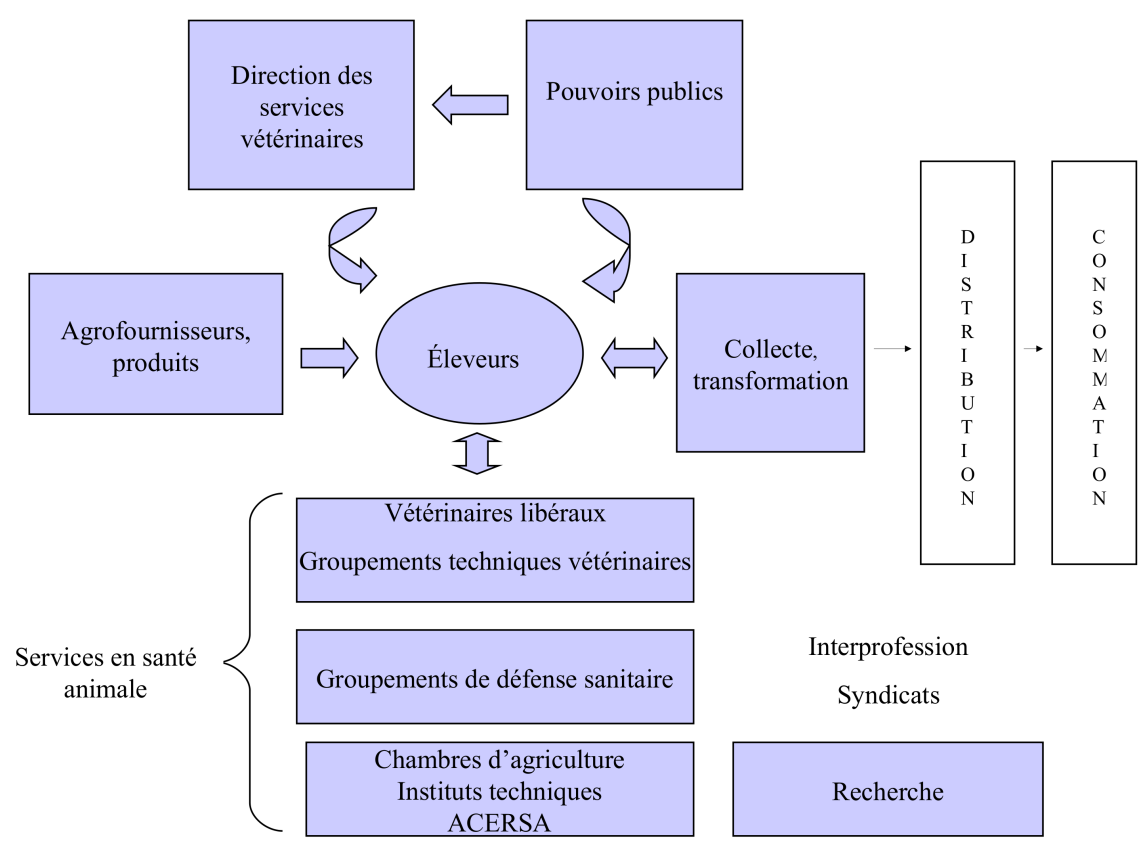

Fig. Les acteurs de la gestion sanitaire des élevages en France.

de gestion du risque sanitaire. Ces entretiens ont permis d'identifier une palette de représentations concernant la santé d'un troupeau (Encadré 2). Quatre grands profils d'éleveurs se sont dégagés, allant d'une attitude active de prévention à une sous-estimation des risques entrâ̂nant des pratiques dangereuses. Les différentes attitudes face au risque sanitaire et les opinions des éleveurs à propos de l'action collective entraînent des attentes très différentes par rapport à une qualification sanitaire. Les résultats de ces enquêtes montrent une grande hétérogénéité du monde de l'élevage. Ils sensibilisent a priori sur la difficulté à faire émerger un consensus en matière de qualification sanitaire. Aussi, le processus de construction collective d'un dispositif de qualification ne peut se mettre en œuvre que dans la durée. Au minimum, il oblige à définir qui a intérêt à participer à cette construction (individus ou structures agricoles), comment assurer l'engagement de chaque participant dans cette construction, quels que soient son statut et la stratégie qu'il poursuit, et comment transformer le débat en prise de décision dans laquelle le plus grand nombre puisse se reconnaître.

\section{Les interactions dans la construction sociale de la qualification sanitaire}

Chacune des approches disciplinaires abordées précédemment a un apport déterminant dans la construction d'une qualification sanitaire. La phase ultérieure consiste à envisager comment associer ces compétences, comment les intégrer dans un projet qui, idéalement, les prendrait en compte sans hiérarchie a priori. Le programme de recherche ACDUQ a mobilisé des chercheurs relevant des sciences nomologiques (zootechnie, médecine vétérinaire, épidémiologie, mathématiques) et des sciences interprétatives (économie, sociologie), mais également des professionnels représentant différents organismes des filières concernées (Groupements de défense sanitaire, Institut de l'élevage). Le dispositif comportait aussi des éleveurs auxquels les différentes enquêtes ont donné la parole $^{10}$. Analyser la façon dont ce collectif a fonctionné illustre la complexité de la mise en œuvre d'une approche pluridisciplinaire dans le contexte de l'élaboration d'une qualification sanitaire.

\section{Construction et « territorialisation »}

Avant d'être le résultat mécanique d'une procédure définie, la décision d'une qualification sanitaire résulte d'une construction sociale collective. Elle n'obéit pas à un seul type de rationalité, scientifique ou juridicoadministrative. Elle mobilise des connaissances savantes et des savoirs empiriques et dépend de nombreuses décisions, lesquelles peuvent engendrer des convergences ou des contradictions. Ces dernières peuvent s'exprimer dans des conflits de conception ou d'intérêt, mais elles peuvent aussi être dépassées à travers des arrangements aux périmètres et aux durées de vie variables. Chacun des acteurs est appelé à revoir son point de vue, parfois à renoncer à une partie de ses intentions ou des objectifs qu'il s'était fixés.

\footnotetext{
${ }^{10}$ Cent vingt éleveurs environ ont été interviewés lors de quatre études distinctes.
} 


\section{Encadré 2. Étude sociologique sur les représentations des éleveurs en matière de santé}

Une étude sur les représentations des éleveurs en matière de santé animale et de risque sanitaire a été conduite en 2007 dans le cadre du projet ACDUQ, à partir d'une quarantaine d'entretiens semi-directifs. L'échantillon des éleveurs bovins retenu a cherché à recouvrir la diversité supposée des situations et des points de vue, incluant des critères liés au type d'exploitation (orientation lait ou viande, fréquence des ventes/achats d'animaux, histoire sanitaire récente, mode de commercialisation) et à la localisation géographique (huit départements présentant des contextes variés en termes de densité d'élevage et d'organisation pour la gestion collective des risques sanitaires). L'analyse a porté sur le métier d'éleveur, sa place dans la société, la santé du troupeau, la connaissance des maladies qui pouvaient l'atteindre, la notion de risque sanitaire, le système de gestion sanitaire français, le rôle des experts et du conseil pour la gestion sanitaire, les pratiques d'élevage centrées sur la prévention ou la lutte contre les maladies contagieuses.

L'enquête a permis d'analyser les représentations que se faisaient les éleveurs de la santé du troupeau (tableau). Par ailleurs, une typologie des attitudes a pu être dégagée, s'appuyant sur les arguments mobilisés par les éleveurs pendant les entretiens et sur les justifications de leurs pratiques dans le domaine de la prévention des maladies contagieuses.

Tableau (extrait). En colonne, quatre types d'éleveurs caractérisés à partir de leur point de vue sur la santé du troupeau.

\begin{tabular}{|c|c|c|c|c|}
\hline $\begin{array}{l}\text { Caractérisation par les } \\
\text { éleveurs de leur point de } \\
\text { vue sur la santé du troupeau }\end{array}$ & $\begin{array}{l}\text { Absence de maladie - } \\
\text { Relations avec le vétérinaire }\end{array}$ & $\begin{array}{l}\text { Regard porté sur } \\
\text { l'apparence des animaux }\end{array}$ & $\begin{array}{l}\text { Pratiques professionnelles } \\
\text { de l'éleveur }\end{array}$ & $\begin{array}{l}\text { Résultats techniques } \\
\text { et économiques }\end{array}$ \\
\hline $\begin{array}{l}\text { Critères mis en avant } \\
\text { par les éleveurs - extraits } \\
\text { des interviews à titre } \\
\text { illustratif }\end{array}$ & $\begin{array}{l}\text { Pas de maladie dépistée } \\
\text { Peu ou pas de frais } \\
\text { vétérinaires }\end{array}$ & $\begin{array}{l}\text { Des animaux de belle } \\
\text { apparence } \\
\text { Un comportement des } \\
\text { animaux } \\
\text { visiblement calme }\end{array}$ & $\begin{array}{l}\text { Des animaux bien logés } \\
\text { et nourris } \\
\text { Un éleveur qui surveille bien } \\
\text { son troupeau }\end{array}$ & $\begin{array}{l}\text { Des résultats technico- } \\
\text { économiques satisfaisants } \\
\text { Pas de désorganisation liée } \\
\text { à la mauvaise santé } \\
\text { du troupeau }\end{array}$ \\
\hline
\end{tabular}

L'adoption d'une décision commune exige l'apparition d'une représentation partagée d'une situation dans laquelle les différents acteurs concernés peuvent être a priori socialement ou juridiquement inégaux ou en désaccord. Dans le cas du travail exposé, aucune représentation collective n'existait a priori. Personne n'a été invité à exposer ses arguments devant une assemblée prête à les entendre au nom d'un problème universellement posé et d'une solution obligatoirement rationnelle à adopter. Au contraire, des jeux de pouvoir structurels se sont manifestés, nourris par les mandats, les rôles et les statuts des acteurs, ou par les influences conjoncturelles, comme l'état des marchés ou l'opinion des consommateurs.

Une hiérarchie implicite des connaissances utilisées et des rôles tenus par les différents acteurs attribuait une valeur sociale aux arguments utilisés selon différents critères : scientifique, économique, sanitaire, juridique, politique. La construction de la qualification peut arriver à terme sans que cette hiérarchie se soit atténuée véritablement, alors même que le dispositif social de la construction s'est autonomisé vis-à-vis de déterminants sociaux plus généraux. Parfois, des crises ont secoué les acteurs impliqués. Elles rappelaient l'effet de cette hiérarchie. Des efforts ont pu être entrepris par tel ou tel acteur afin d'y échapper. Ils évoquaient l'abandon de positions prises habituellement hors du processus de construction.

On était donc en présence d'une «territorialisation » (Deleuze et Guattari, 1980) initiale du processus de construction de connaissances concernant la qualification sanitaire. Cet «agencement » (ibidem) a pris la forme d'une organisation implicite des connaissances et des rôles de chacun. Elle s'est imposée à travers les codes de raisonnement et de production de connaissances de chacune des disciplines convoquées. La « déterritorialisation », constatée au fur et à mesure de l'avancée du programme, a consisté à décoder le fonctionnement du groupe de recherche et à expliciter et évaluer la pertinence d'une hiérarchie implicite des disciplines convoquées, des méthodes utilisées et des flux de connaissances convoquées et produites.

La simple distribution des critères que sont la définition des maladies, les zones dans lesquelles elles se manifestent, les statuts et les rôles sociaux des acteurs concernés, dessine le périmètre de la construction de la décision concernant l'adoption ou non d'une qualification. Mais chaque critère reste porteur de traits socioculturels qui le rattachent à des dimensions directement ou indirectement actives dans l'élaboration de la construction de la décision de qualification. Par exemple, les distinctions géographiques des zones d'élevage recouvrent l'existence de pratiques d'élevage dont chacun considérera, sans d'ailleurs toujours pouvoir le prouver, qu'elles participent à la propagation, à la prévention ou à l'éradication des maladies. Les pratiques professionnelles d'un éleveur breton restent différentes, ne serait-ce qu'anthropologiquement, de celles d'un éleveur bourguignon ou danois. Au-delà des spécificités géomorphologiques de tel ou tel territoire, un sentiment d'appartenance ou d'identification à tel ou tel groupe professionnel alimentera des pratiques locales, qui développeront des formes d'individualisme ou de solidarité et joueront sur les prises de décision en matière de qualification sanitaire. La "déterritorialisation » évoquée plus haut a eu pour effet de substituer aux effets de détermination spatiale et culturelle des formes d'engagements volontaires «hors sol », influencées par les seules interactions des acteurs. Ces formes se manifestent au-delà 
de l'entourage (Goetz, 2007). Elles transcendent, sans les faire disparaître, les cultures traditionnelles de milieu ou de terroir. Nous parlerons dans ce cas de «reterritorialisation » du processus de production de connaissances engagé par le groupe de recherche.

\section{Épistémologie et société}

Le programme ACDUQ a rassemblé des scientifiques plutôt tenants de la démarche expérimentale, d'autres « condamnés » à interpréter le monde, d'autres, enfin, attachés avant tout aux connaissances empiriques. Cette collaboration a reproduit un théâtre social à la pression duquel aucun des acteurs n'a pu véritablement se libérer, malgré la dynamique de « territorialisation » ou « d'agencement » évoquée.

La démarche expérimentale repose, comme la modélisation, sur la nécessité d'une réduction du réel supposé auquel elle s'applique. Ses adeptes sont parfois restés sceptiques quant à la qualité des connaissances empiriques. De même, les interprétants ont parfois suspecté l'irréalisme d'une démarche « réductionniste » qui se serait contentée de quelques éléments d'un réel trop complexe pour être appréhendé dans son ensemble. Les épidémiologistes ont cherché à proposer des connaissances prédictives capables d'orienter une prise de décision leur semblant raisonnable. La pertinence des éléments choisis pour constituer les modèles proposés a été évaluée au titre de la capacité que chacun pouvait avoir à rendre compte d'un réel supposé (Pécaud, 2005).

La recherche a montré que la valeur sociale des résultats d'ensemble ne pouvait être estimée au seul regard de la valeur académique des résultats scientifiques, surtout quand cette dernière restait définie au sein de la seule sphère des sciences nomologiques. D'une part, l'interdisciplinarité attachée à la réalisation du programme a rendu nécessaire d'admettre une pluralité des valeurs des résultats scientifiques. Le travail interdisciplinaire visait l'émergence de connaissances nouvelles par rapprochement disciplinaire et prise en compte de la complexité $q^{\prime} u^{\prime} i l$ engendrait de fait. Quelques avancées peuvent être signalées : réflexion sur la pertinence sociale des modèles épidémiologiques et économiques; valorisation des connaissances empiriques comme déterminants de l'action; pluralité des représentations au regard des prises de décision. Elles n'établissent qu'en partie l'égalité du rôle de chacune des disciplines dans la production des connaissances disciplinaires produites. D'autre part, l'évaluation du programme ne peut porter sur ses seuls résultats finaux; elle se doit d'être alimentée par une réflexion sur l'évaluation des conditions de travail en commun ayant présidé à la formation du processus de recherche lui-même. Parmi les résultats qui ne peuvent être négligés, on peut noter la compréhension réciproque des savoirs, la production de connaissances par émergence, la construction de la confiance entre chercheurs.

\section{Discussion et conclusion}

À l'issue de ce tour d'horizon sur le rôle et la place de chaque discipline dans la construction d'une qualification sanitaire, il est frappant de constater le rôle central que joue l'usage disciplinaire de la notion de risque. Cette notion est omniprésente. Elle imprègne chaque raisonnement, alors même qu'elle n'a fait l'objet d'aucune discussion particulière. Chacun l'aborde de son point de vue et selon sa propre acception. Si la construction fut possible, c'est au titre de la constitution d'un véritable " cercle herméneutique » (Schleiermacher, 1987) que l'usage implicite de la notion de risque a mis en œuvre. La multiplicité des définitions du risque et de leur interprétation a rendu compréhensible l'ensemble de la démarche, qui, elle-même, n'est devenue compréhensible que du fait de cette multiplicité.

Le biologiste met en avant les nombreuses incertitudes biologiques, notamment sur le devenir des agents infectieux dans l'organisme de l'animal et le mode de propagation des maladies entre animaux. Par ailleurs, les tests de dépistage étant imparfaits, il le traduit généralement par deux notions de risque : celui de ne pas détecter un animal infecté, qu'on qualifie par un défaut de sensibilité ${ }^{11}$ de la technique de dépistage; celui de croire à tort qu'un animal est infecté, soit un défaut de spécificité ${ }^{12}$.

Le recours aux mathématiques permet alors d'introduire une approche probabiliste de ces incertitudes et d'intégrer - associer ensemble - les connaissances biologiques et leurs incertitudes grâce à la modélisation. Les modèles développés permettent alors de quantifier deux autres notions de risque qui découlent de ces travaux. Comme il a été vu précédemment, le risque acheteur estime la probabilité qu'un élevage qualifié indemne soit en fait infecté; il renvoie à la même notion générale que le défaut de sensibilité, cette fois appliqué au dispositif de qualification quand il ne détecte pas des troupeaux infectés. Le risque vendeur, quant à lui, estime la probabilité de refuser la qualification à un élevage pourtant indemne, et renvoie donc à un défaut de spécificité. Finalement, les approches statistiques et de modélisation mettent à la disposition des acteurs concernés des outils qui leur permettent, en vue de leurs prises de décisions, de mettre en balance risque vendeur, risque acheteur et coûts des dispositifs envisagés.

\footnotetext{
11 La sensibilité d'un test mesure la proportion d'animaux infectés que le test détecte positifs.

12 La spécificité d'un test mesure la proportion d'animaux indemnes que le test détecte négatifs.
} 
Le point de vue économique introduit pour sa part une balance entre risque $d^{\prime}$ ' acheter » l'infection et coût, d'un côté, ou bénéfice de maîtriser ce risque, de l'autre, ce qui renvoie aussi à une approche probabiliste du risque. La composante sociologique replace enfin l'ensemble de ces notions dans un contexte social, en ajoutant deux composantes essentielles à la construction de la qualification. La première est que risques, coûts, bénéfices escomptés et intérêts pour une qualification sanitaire diffèrent énormément selon les acteurs impliqués, de par leur positionnement institutionnel et leur activité économique. La deuxième se réfère à la variabilité des perceptions des éleveurs vis-à-vis des risques sanitaires et aux représentations qu'ils ont d'un troupeau sain. Ainsi, l'approche pluridisciplinaire mise en œuvre permet de travailler de manière approfondie sur cette notion de risque en la captant de plusieurs points de vue complémentaires, enrichissant par là même le processus de construction de la qualification sanitaire.

Mettre en œuvre une qualification sanitaire vis-à-vis $\mathrm{d}$ 'une maladie infectieuse répond au besoin de favoriser les échanges commerciaux entre troupeaux, tout en garantissant les acheteurs contre l'introduction dans leur cheptel de maladies importantes du point de vue de la santé animale ou publique. Partant de cette idée simple, il s'avère que décider de l'importance stratégique d'une qualification n'est ni immédiat ni univoque. Les acteurs peuvent exprimer des points de vue et des intérêts divergents, techniques, économiques ou éthiques, dans un contexte où une qualification sanitaire peut avoir des impacts économiques majeurs. À cela s'ajoute la variété des représentations du risque sanitaire qui fait que tous les acteurs ne sont pas forcément prêts à entrer dans un tel processus. Les approches économiques et sociologiques apportent un éclairage déterminant sur ces composantes. Le projet ACDUQ a donné lieu à différents constats esquissant la dimension d'une coconstruction en matière de qualification sanitaire. Le premier d'entre eux a porté sur l'influence des administrations, des filières professionnelles, des vétérinaires de terrain et des groupements d'éleveurs en matière de qualification sanitaire. Le deuxième a concerné la formation de la confiance dès qu'ont été confrontées connaissances scientifiques diverses et pratiques professionnelles en vue de l'élaboration d'une décision. Le troisième a eu trait à l'influence des informations et de leur accessibilité sur la formation des convictions et sur l'orientation des décisions individuelles. Le dernier constat a porté sur le poids des habitudes culturelles des éleveurs au regard de nouveaux engagements professionnels qui pouvaient les mener, dans un premier temps, à une "déterritorialisation » critique de leurs pratiques et, dans un second, à l'éventuelle « reterritorialisation » acceptée de celles-ci.

Ces différents constats ouvrent autant de perspectives. Le premier pose les conditions de réussite de la coconstruction. Elle ne peut se centrer sur la seule validité des connaissances scientifiques utilisées, mais doit tenir compte des formes de leur réception sociale dans des dimensions politique, technique, administrative, juridique. Le deuxième invite à rejeter des schémas trop simplistes de prise de décision, dans lesquels l'orientation de l'activité aurait pour cause la connaissance. La décision ne dépend pas de la seule connaissance, fût-elle scientifiquement valide. Elle naît dans des circonstances aux caractères multiples. Le pouvoir des connaissances qui l'éclairent, la puissance des arguments qui cherchent à l'orienter dépendent de la confiance que les acteurs attribuent à ceux qui les produisent. Le troisième constat met en avant l'importance de la compréhension réciproque des différents acteurs. La communication entre les différents acteurs impliqués dans la coconstruction ne peut se réduire à de simples traductions de discours d'« experts ». Elle est aussi délibération. Quant au dernier constat, il oblige à prendre en compte les représentations des éleveurs en matière de santé animale, mais aussi leurs exigences. Il nécessite leur mobilisation à part entière dans le projet de recherche.

\section{Remerciements}

Ce travail a été conduit avec le support financier de l'Agence nationale de la recherche (ANR), des établissements de recherche (Inra, Cirad, Cemagref, Ifremer) et de l'ADEME, impliqués au sein du programme Agriculture et développement durable, projet ANR-05-PADD-014, ACDUQ.

\section{Références}

Cameron, A.R., Baldock, F.C., 1998. A new probability formula for surveys to substantiate freedom from disease, Preventive Veterinary Medicine, 34, 1-17.

Cannon, R.M., Roe, R.T., 1982. Livestock Disease Surveys: A Field Manual for Veterinarians, Canberra, Australian Government Publishing Service.

Centner, T.-J., Wetzstein, M.-E., 1987. Reducing moral hazard associated with implied warranties of animal health, American Journal of Agricultural Economics, 69, 1, 143-150.

Chymis, A.G., James, H.S., Konduru, S., Pierce, V.L., Larson, R.L., 2007. Asymmetric information in cattle auctions: The problem of revaccinations, Agricultural Economics, 36, 1, 79-88.

Deleuze, G., Guattari, F., 1980. Mille plateaux, Paris, Les Éditions de Minuit.

Domenech, J., Lubroth, J., Eddi, C., Martin, V., Roger, F., 2006. Regional and international approaches on prevention and control of animal transboundary and emerging diseases, Annals of the New York Academy of Sciences, 1081, 90-107.

Durand, B., Martinez, M.-J., Calavas, D., Ducrot, C., 2009. Comparison of strategies for substantiating freedom from scrapie in a sheep flock, BMC Veterinary Research, 5, 16. 
Ezanno, P., 2006. Rapport technique sur le modèle stochastique à compartiments en temps discret de propagation du virus de la maladie des muqueuses (BVDV) dans un troupeau bovin laitier. INRA ENV Nantes, Nantes.

Ezanno, P., Fourichon, C., Viet, A.-F., Seegers, H., 2007. Sensitivity analysis to identify key-parameters in modelling the spread of bovine viral diarrhoea virus in a dairy herd, Preventive Veterinary Medicine, 80, 1, 49-64.

Ezanno, P., Fourichon, C., Seegers, H., 2008. Effect of herd structure and type of virus introduction on the spread of bovine viral diarrhoea virus (BVDV) within a dairy herd, Veterinary Research, 39, 39.

Goetz, B., 2007. L'araignée, le lézard et la tique : Deleuze et Heidegger lecteurs de Uexkül, Le Portique, 20, 110-132.

Johnson, W.O., Su, C.-L., Gardner, I.A., Christensen, R., 2003. Sample size calculations for surveys to substantiate freedom of populations from infectious agents, Biometrics, 60, 165-171.

Kling-Éveillard, F., 2001. L'Étude de motivation pour prendre en compte les points de vue des éleveurs, Paris, Institut de l'élevage.

Martinez, M-J., Durand, B., Calavas, D., Ducrot, C., 2010. Methodological approach for substantiating disease freedom in a heterogeneous population. Application to ovine scrapie, a disease with a strong genetic susceptibility, Preventive Veterinary Medicine, 95, 1-2, 108-114.

Pécaud, D., 2005. Risques et précautions, Paris, La Dispute.
Rat-Aspert, O., Weldegebriel, H.T., Stott, A.W., Fourichon, C., 2008. Managing animal health status information in the cattle market, 12th Congress of the European Association of Agricultural Economists, August 26-29, 2008, Ghent, Belgium (http://ageconsearch.umn.edu/handle/44064).

Schleiermacher, F., 1987. Herméneutique, Genève, Labor et Fides.

Toma, B., Dufour, B., Sanaa, M., Bénet, J.-J., Shaw, A., Moutou, F., Louzà, A., 1996. Épidémiologie appliquée à la lutte collective contre les maladies animales transmissibles majeures, MaisonsAlfort, AEEMA.

Viet, A.-F., 2008. Rapport technique sur le modèle stochastique individu-centré BVDSim de propagation du virus de la maladie des muqueuses (BVDV) dans un troupeau bovin laitier ou allaitant. INRA ENV Nantes, Nantes

Viet, A.-F., Fourichon, C., Seegers, H., Jacob, C., GuihenneucJouyaux, C., 2004. A model of the spread of the bovine viraldiarrhoea virus within a dairy herd, Preventive Veterinary Medicine, 63, 3-4, 211-236.

Wolf, C., 2005. Producer livestock disease management incentives and decisions, International Food and Agribusiness Management Review, 8, 1, 46-61.

Zepeda, C., Salman, M., Thiermann, A., Kellar, J., Rojas, H., Willeberg, P., 2005. The role of veterinary epidemiology and veterinary services in complying with the World Trade Organization SPS agreement, Preventive Veterinary Medicine, $67,125-140$.

Reçu le 13 février 2009. Accepté le 1er octobre 2009. 